Meta

Journal des traducteurs

Translators' Journal

\title{
Traduction, fertilisation et internationalisation : les calques en espagnol
}

\section{J. C. Santoyo}

Volume 32, numéro 3, septembre 1987

La fertilisation terminologique dans les langues romanes

URI : https://id.erudit.org/iderudit/004029ar

DOI : https://doi.org/10.7202/004029ar

Aller au sommaire du numéro

Éditeur(s)

Les Presses de l'Université de Montréal

ISSN

0026-0452 (imprimé)

1492-1421 (numérique)

Découvrir la revue

Citer cet article

Santoyo, J. C. (1987). Traduction, fertilisation et internationalisation : les calques en espagnol. Meta, 32(3), 240-249. https://doi.org/10.7202/004029ar d'utilisation que vous pouvez consulter en ligne. 


\title{
TRADUCTION, FERTILISATION ET INTERNATIONALISATION : LES CALQUES EN ESPAGNOL
}

\author{
Julio CESAR SANTOYo \\ Université de León, Espagne
}

Comme les autres langues de la culture dite atlantique, ou culture occidentale (français, portugais, catalan, anglais, italien...), l'espagnol, qu'on le veuille ou non, est en train de se convertir rapidement en une langue traduite. Et je ne parle pas en ce moment des 8000 traductions qui sont publiées chaque année dans mon pays, la moitié d'entre elles étant des versions de l'anglais, 1800 du français, 700 ou 800 de l'italien et de l'allemand, etc. Je fais plutôt allusion au fait qu'en espagnol, tout comme dans ces autres langues avec lesquelles il se partage la moitié de la planète, il est en train de se développer un abondant corpus lexical et locutionnel, traduit précisément de ces autres langues qui l'accompagnent dans l'Histoire. Si jusqu'à présent, les théoriciens de la science translémique ont étudié la traduction en tant que processus de transvasement linguistique ou bien comme son résultat textuel, peut-être devront-ils aussi commencer à la considérer comme l'un des facteurs les plus puissants de la fertilisation et internationalisation linguistique.

En plus des ressources propres à chaque langue, les lexicologues et terminologues ont presque toujours porté leur attention, en ce qui concerne la formation d'unités terminologiques de tout type, sur ce que Haugen, Chansou et Klajn appellent emprunts intégraux, ceux dans lesquels (comme robot, sandwich, spaghetti ou whisky), "la forme étrangère est entièrement adoptée par la langue emprunteuse; il n'y a donc aucune substitution de forme $» 1$. Et, en particulier, en ce qui concerne le français et l'espagnol, il y a déjà plusieurs dizaines d'années qu'un tel intérêt est porté sur les emprunts intégraux d'origine anglo-saxonne. La situation, cependant, ne change pas dans d'autres langues, parmi lesquelles l'anglais lui-même : rappelons-nous à ce sujet le volume de Mary S. Serjeantson $A$ History of Foreign Words in English, publié en 1935.

Les analyses les plus sommaires, nonobstant, indiquent que, en plus de ce type d'emprunts étrangers, les langues romanes en particulier (de même que l'anglais, que sans tomber dans l'hétérodoxie nous pourrions considérer comme semi-roman) ont commencé il y a quelques années à utiliser massivement ce qu'on appelle le calque, phénomène encore peu étudié, et non seulement en français, en anglais ou en espagnol, mais aussi dans de nombreuses autres langues, européennes ou non. Il y a quatorze ans, par exemple, et parlant à la fois du croate, du slovène, du serbe, du tchèque et du slovaque, Josip Jernej pouvait affirmer que

se per le lingue di tutte queste popolazioni esistono studi importanti sull'elemento lessicale forestiero..., molto resta da fare in questo campo per $i$ calchi...2

Dans une certaine mesure, nous ne devons pas nous étonner de cette pauvreté d'études, étant donné que la notion linguistique même de calque est relativement récente, de même que sa dénomination explicite. Si le terme emprunt est encore jeune dans son acception linguistique, on ne sera pas surpris que ni calque ni loan-translation ne figurent dans le Oxford English Dictionary (y compris le Supplément), ni que le Diction-

Meta, $X X X I I, 3,1987$ 
naire de l'Académie royale espagnole n'ait inclus le mot jusqu'à sa dernière édition de 1934. Tout cela, bien qu'en 1912 déjà Sandfeld ait publié à Leipzig ses Notes sur les calques linguistiques.

Malgré tout, on a souvent débattu, ces dernières années, de la nature du calque, ses catégories et formes, avec certains désaccords quant à la portée exacte du terme. Des noms comme ceux de Deanovic (1934), Migliorini (1948), Hristea (1967), Guiraud (1971), Jernej (1973), Giraud (1978), Chansou (1984) et Montes-Giraldo (1985) sont autant de références obligatoires dans la révision du problème de différentes nationalités. Les discussions, cependant, aussi bien terminologiques que conceptuelles, ont un dénominateur commun minimum accepté par tous : le calque implique toujours traduction. " (It is) a compound, derivative or phrase brought into one language THROUGH TRANSLATION of the constituent parts of the term in another language 》 (Mario Pei : 1966) ; tout " consiste à TRADUIRE la forme étrangère par son équivalent indigène " (Guiraud : 1971); le Dictionnaire espagnol de l'Académie définit ce sens comme "l'adaptation d'un mot étranger, par la TRADUCTION de sa signification complète ou de celle de chacun des éléments le composant "; à leur tour, les auteurs du Dictionnaire de linguistique (Jean Dubois et al.) délimitent ainsi le terrain : "On dit qu'il y a calque linguistique quand, pour dénommer une notion ou un objet nouveau, une langue (a) TRADUIT un mot, simple ou composé, appartenant à une langue (b) en un mot simple ou en un terme composé formé de mots existant aussi dans la langue. " Enfin, "l'emprunt (écrit García-Yebra) aussi bien naturalisé que dans la forme originale (mot étranger) n'est pas un procédé de traduction, mais précisément la renonciation à la traduction : LE CALQUE, LUI, EST TRADUCTION ${ }^{3}$. Non seulement il est traduction, mais même Vinay et Darbelnet, dans leur célèbre Stylistique comparée du français et de l'anglais, le qualifient de second procédé technique de traduction, dans lequel - disentils - " on emprunte à la langue étrangère le syntagme, mais on TRADUIT littéralement les éléments qui le composent ${ }^{4}$. La confirmation ultime de cette nature traduite, nous pourrions la trouver dans la dénomination sous laquelle on le connaît en anglais : loan-translation.

Cela dit, la notion de calque s'est traditionnellement limitée aux unités syntagmatiques que Jean Giraud a appelées néologismes notionnels, « ainsi appelés parce qu'ils ne concernent pas un mot, mais l'expression d'une idée à travers une association inédite de termes $\%$. Implicitement ou explicitement, nombreux sont ceux qui adhèrent à cette même considération. Wilss montre comme exemples de calques de l'anglais les termes allemands Geburtenkontrolle, Schaugeschäft ou Familienplanung, traduits selon cette méthode des originaux anglo-saxons birth control, show business et family planning. J'ai moi-même considéré en plus d'une occasion que la nature du calque se limitait exclusivement à ces néologismes notionnels. Il y a trois ans, par exemple, lors d'une table ronde de la Fondation Alphonse X Le Sage à la Bibliothèque nationale de Madrid qui portait sur 'Quelques aspects théoriques de la traduction', entre autres celui des calques comme procédé de traduction, je me rappelle avoir dit, suivant la ligne de pensée de Giraud :

Jamais la traduction d'une association normale d'idées ne constitue un calque. C'est le cas, par exemple, de l'expression française " avec la porte ouverte ", qui ne constitue en aucune façon un calque lorsqu'on le traduit en espagnol " con la puerta abierta". Le calque implique, par conséquent, une association lexicale et notionnelle inédite dans la propre langue d'origine, une association en quelque sorte " anormale ", avec d'abondants éléments métaphoriques ou antithétiques, et il semble par la suite choquant, surprenant...,

comme peuvent l'être les calques français de l'anglais rideau de fer et gratte-ciel. 
Aujourd'hui, trois ans après ces mots, ayant reconsidéré plusieurs fois le problème de la nature, de la condition et des types de calques, je dois dire que tous ceux qui ont consacré un peu ou beaucoup de temps à cette étude ont peut-être péché d'une grande étroitesse de vue. Aveuglés par les feux d'artifice de ces brillantes métaphores du langage quotidien, comme le sont fuite de cerveaux, mont-de-piété ou soucoupe volante, nous nous sommes limités à ce type particulier de syntagmes, laissant de côté d'autres calques, sans doute aussi nombreux, sinon plus que ceux déjà cités. Si nous repassons les définitions qui ont été proposées jusqu'à présent, nous ne trouverons pas dans aucune d'elles que la "matière " originale sujette à calque est, de quelque façon que ce soit, prédéterminée. On parle, oui, de "forme étrangère » et de "mot étranger " (Guiraud), de "schema preesistente" (Jernej), de " concepto creado en otra lengua " (Montes-Giraldo), etc. Barbara Strang le considère seulement comme " a complex form in the sourcelanguage ", Michel Chansou comme " un modèle étranger " ou comme une " unité lexicale", et Wilss comme "morphologically analizable source-language syntagms (primarily noun compounds and adjective-noun-collocations" 6 . Personne ne semble donc considérer qu'a priori les sources de possibles calques doivent être d'une condition sémantique déterminée ou d'une autre, doivent réunir telle ou telle exigence " notionnelle ». " $A$ las cinco de la tarde », équivalent direct de "à cinq heures ", n'est en espagnol qu'une expression banale, nullement métaphorique, diamétralement opposée à ce qui pourrait être considéré un néologisme notionnel, une association lexicale inédite, etc. Quand le vendredi 16 mai 1986 le Corriere della Sera, journal de Milan, rendait compte de la mort du pilote de Formule 1 Elio de Angelis dans un accident d'automobile, la chronique se terminait sur cette phrase : "Il destino se l'è portato via, $A L L E C I N$ QUE DELLA SERA, come un 'matador' sfortunato "7. Il n'y a pas de doute que l'expression italienne dans ce contexte déterminé est un calque évident de l'usage espagnol renforcé - comme si cela ne suffisait pas - par l'allusion au "matador".

Je crois donc qu'il est temps d'admettre que la notion de calque a des aires d'incidence notablement plus étendues et diverses que ce qui était reconnu. Considérons comme valide, en effet, une des définitions, celle de Strang :

A loan-translation (écrit-elle) is represented by a type, usually derived from a complex form in the source-language, in which the elements are rendered into corresponding ones in the borrowing language; there is no outer similarity of form, but the structure and function are alike $e^{8}$.

La déduction immédiate est qu'en stricte application opérative, le procédé traducteur ici mentionné comme générateur de calques, tout comme les conditions du résultat final, sont applicables sans réserves à un corpus considérablement riche de lexèmes, syntagmes et locutions, tous calqués (c'est-à-dire traduits) sur des formes similaires préalables dans une autre langue. Partant, on doit considérer comme calques :

1. Ceux qui dérivent d'une unité conceptuelle de référence générique, comme produit national brut, calque de l'anglais gross national product, et origine à son tour de l'espagnol producto nacional bruto. En espagnol impuesto sabre el valor añdido (le célèbre $I V A$ ) est une forme calquée de la taxe sur la valeur ajoutée française. De la même manière sindrome de immunodeficiencia adquirida, le redoutable SIDA, est un calque de l'anglais, peut-être par le biais de syndrome d'immunodéficience acquise français. Dans ces cas-là et dans d'autres centaines d'entre ceux que présente l'actualité, scientifique ou non, on remarque un processus direct de traduction, pratiquement toujours littéral. Ces calques sont précisément, comme le dit Chansou, ceux qui facilitent énormément " la communication internationale dans le domaine scientifique et technique, dans la mesure 
où des structures lexicales identiques introduites dans plusieurs langues peuvent être aisément reconnues par les spécialistes $"$.

2. On doit également considérer comme calques ceux qui, conformément à ce qui a été dit, reproduisent dans la langue de traduction une unité conceptuelle de référence spécifique. Il est évident qu'uniquement à travers un processus de traduction, et uniquement à son tour en version traduite, sont connus aujourd'hui dans les diverses langues les équivalents de Croix rouge, Maison blanche, Sixième flotte, etc., correspondant aux formes espagnoles Cruz Roja, Casa Blanca, Sexcta Flota..., ou aux italiennes Croce Rossa, Casa Bianca, etc. (Personne, sauf peut-être le plus snob des pédants, n'a l'idée en parlant en français, en italien ou en espagnol, d'introduire dans la conversation des expressions du type Red Cross ou White House; parce que dans ce groupe de calques, de même que dans ceux du paragraphe antérieur, la traduction est péremptoirement nécessaire et pratiquement automatique.) Ce n'est qu'exceptionnellement, et répondant à l'idiosyncrasie particulière de chaque langue, que ces "noms propres" (ils se comportent comme tels) rejettent le calque et conservent leur forme originale également dans la deuxième langue. En espagnol, par exemple, nous n'avons pas traduit le nom de la montagne la plus haute de France, le Mont-Blanc, de même que les Anglais ne l'ont pas fait non plus, et cela bien que ses constituants lexicaux soient diaphanes. Au contraire, les Portugais et les Italiens l'ont traduit, Monte Branco et Monte Bianco, langues dans lesquelles il apparaît ainsi comme calque de la dénomination française.

3. Nous prendrons en compte, enfin, ceux dont la matière originale est une unité conceptuelle à connotation métaphorique, et qui forment, de fait, la plus grande part de ceux qui jusqu'à présent ont été improprement considérés comme seuls calques. Ici nous pouvons citer des exemples tels que ceux représentés en français par le chant du cygne ou vision du monde, qui viennent de l'allemand, ou comme l'homme de la rue, homme grenouille ou liste noire, calqués de l'anglais. Ce troisième groupe n'exclut en aucun moment les calques de caractère scientifique, dans lesquels la connotation métaphorique peut également être présente : ainsi aqua pesada et lluvia acida en espagnol, eau lourde et pluie acide en français, qui calquent l'anglais heavy water et acid rain. $\grave{A}$ ce groupe, d'ailleurs, appartiennent en général les calques monolexématiques. Il y a des auteurs qui attribuent au calque une condition syntagmatique innée. Mais il existe également, entre les diverses langues, de très nombreux calques de significations étrangères (significations de caractère presque toujours métaphorique), qui s'accumulent sur des noms, des verbes ou des adjectifs préexistants dans la langue de traduction. De même que dans les calques syntagmatiques, sur un support formel préalable, mais dans ce cas monolexématique, on applique un concept nouveau pour la langue qui le reçoit. Telle est la condition des nouvelles significations acquises par les mots espagnols plataforme (politique, revendicative, etc.), peinar (fouiller à fond), lectura (révision, interprétation), traduire (convertir, se concrétiser, prendre forme), escalada (augmentation spectaculaire), emergencia (urgence), etc., dérivés de quelques-unes des acceptions anglaises de platform, to comb, reading, to translate, escalation et emergency.

Ayant donc établi les paramètres qui, à mon avis, délimitent la nature et les variantes du calque, je passe à un deuxième chapitre de mon exposé : son incidence dans l'espagnol actuel en particulier et dans le reste des autres langues romanes en général.

En l'absence d'études diachroniques complètes, il ne semble pas aujourd'hui qu'il ait été un phénomène très fréquent dans le passé de nos langues. Excepté certains moments et points concrets de diglossie et de bilinguisme, l'histoire des langues occidenta- 
les en présente des exemples plutôt rares jusqu'à ce siècle-ci. En ce qui concerne l'anglais, Strang a pu dire dans $A$ History of English que

borrowings of this type have rarely been as frequent as other types of innovation in English, and are thinly represented in the last two centuries, though one familiar example is "Superman ", formed by G.B. Shaw on the model of Nietzsche's "Übermensch »10.

La même chose se passe en espagnol : l'académicien Rafael Lapesa consacre quelques lignes bien succinctes aux calques dans les sept cents pages que compte sa dernière édition de la Historia de la Lengua Española (1983).

Face à cette situation, ce que mon intervention prétend mettre en évidence est, précisément, l'énorme développement qu'a acquis durant ces dernières années ce phénomène linguistique, déjà converti en l'un des principaux moteurs de l'actuel changement lexical, syntagmatique et locutionnel. Il faut dire aussi que le calque est en train de s'avérer l'un des facteurs les plus dynamiques, perméables et réceptifs de la langue, avec un fort impact et une extrême abondance dans le parler et l'écrire quotidiens, où l'on compte déjà par de nombreuses centaines (centaines, j'insiste) les exemples de semblables microtextes traduits. Me limitant seulement au troisième groupe, c'est-à-dire aux calques à connotation métaphorique, je pourrais vous donner une longue liste, qui épuiserait rapidement votre patience. Je préfère vous renvoyer à l'appendice et être ici plus bref. Voici, nonobstant, quelques échantillons.

$\begin{array}{ll}\text { estacion de servicio } & \text { cafe teatro } \\ \text { ciudad dormitorio } & \text { balanza de pagos } \\ \text { caja negra } & \text { nueva ola } \\ \text { reaccion en cadena } & \text { septimo continente } \\ \text { monte de piedad } & \text { caballo de vapor } \\ \text { paso cebra } & \text { carta verde } \\ \text { retrato robot } & \text { relaciones publicas } \\ \text { alta costura } & \text { grupo de presion } \\ \text { mesa redonda } & \text { fin de semana } \\ \text { conferencia de prensa } & \text { control de la natalidad } \\ \text { otoño caliente } & \text { esqui nautico } \\ \text { cortina de humo } & \text { puesta a punto } \\ \text { lista de bodas } & \text { mercado negro } \\ \text { hombre de letras } & \text { bebe probeta } \\ \text { primera dama } & \text { razon de estado } \\ \text { cinturon de seguridad } & \text { pleno empleo } \\ \text { lavado de cerebro } & \text { lista negra } \\ \text { goma de mascar } & \text { marea negra } \\ \text { alta fidelidad } & \text { musica de camara.. }\end{array}$

Faut-il poursuivre ? Je pourrais continuer cette litanie durant plus d'une heure. Je ne crois pas être trop près de l'exagération si j'affirme que nous commençons déjà, en espagnol, à parler en clef de calques. Et d'après ce que je constate, le même phénomène est en train de se produire dans le reste des langues d'Occident. J'ai dit au début de mon intervention qu'il semble que nos langues soient en passe de se convertir en langues " traduites ". Parce qu'en cela l'espagnol ne fait pas montre d'un comportement spécial : il est seulement un témoin de plus d'un très fort mouvement d'internationalisation d'unités terminologiques, particulièrement commun, il faut le dire, dans les langues romanes. Si de l'espagnol je me tourne vers l'italien, par exemple (et ne parlons pas du portugais), le panorama est étonnamment le même, comme s'il s'agissait non plus de langues sours, mais de langues jumelles. Un seul exemplaire du Corriere delle Sera, du 16 mai 1986 déjà cité, nous offre, entre autres, fine settimana, plattaforma, opinione pubblica, conferenza stampa, pellerossa, vertice, controllo degli armamenti, fuga in avanti, lungometraggio, campo di concentramento, messa in scena, commedia musicale... 
Une chose peut être déduite presque immédiatement du rapprochement multilingue de nos calques, à savoir que les langues romanes dépendent ici étroitement les unes des autres et individuellement de la langue française. Si nos emprunts lexicaux montrent aujourd'hui, dans un pourcentage qui atteint les quatre-vingts pour cent, une provenance majoritairement anglo-saxonne, l'italien, l'espagnol, le portugais, etc., continuent à réaliser la plupart de leurs calques sur le français, aussi bien s'il s'agit d'une expression propre à cette langue que si c'est chez elle, à son tour, un calque d'une autre langue.

En termes généraux, et avec la réserve qu'impose toute généralisation, le processus habituel qu'observe aujourd'hui le calque dans les aires romanes suit ces pas détaillés. 1) Naissance ou existence préalable dans l'une de nos langues d'une unité terminologique déterminée ; comme alternative, création ou existence préalable de l'unité dans une langue non romane, fréquemment l'anglais ou l'allemand. 2) Dans ce dernier cas, traduction " tentative " de ladite unité au français, consolidation et usage courant dans cette langue. 3) Fertilisation du français sur le reste des langues romanes et traduction dans celles-ci de la forme française. 4) Internationalisation de l'expression dans le monde roman et fertilisation à partir de celui-ci sur d'autres zones linguistiques géographiquement proches : grec, polonais, basque, hollandais, etc. 5) Circulation internationale généralisée du terme, "comme fait de civilisation imposé par la réalité à la fin du $\mathrm{XX}^{e}$ siècle $» 11$.

Ce phénomène n'exclut pas que des processus parallèles, ou même identiques, puissent se produire dans d'autres zones idiomatiques. Prenons un cas paradigmatique : celui de l'expression anglaise sleeping-car. Dans l'exemplaire du 5 janvier 1839 on lisait déjà dans le Mechanic's Magazine (p. 240) : "The introduction of the newly-invented sleeping-cars on our railroads... " En 1875, on voit apparaître la traduction française, qui représente un calque légèrement modifié : wagon-lit ; et à partir du français, par un système identique de calque, il passe à l'italien (vagone-letto), au catalan (vago-llit) et à l'espagnol (coche-cama). Dans tous ces cas, se répète le modèle "syntactique " français : d'abord le véhicule (wagon, coche, carro, vagone...), ensuite, accolé à lui, le plus caractéristique de ses composants : lit, letto, cama, llit... Mais tandis que cette vague concrète de fertilisation et d'internationalisation s'épandait à partir du français à travers le sud de l'Europe, arrivant même jusqu'au grec $\kappa \lambda \iota v \alpha-v \alpha \xi \alpha$ (de $\kappa \lambda \iota \nu \eta$, lit et auava, voiture, wagon), une autre vague distincte de calques ayant la même origine couvrait les aires linguistiques du nord du continent avec une traduction beaucoup plus littérale de l'original, au moyen d'un premier indicateur de fonction (" dormir ") et d'un second indicateur de véhicule : tel est le cas du hollandais slaap-wagen, de l'allemand Schlaf-wagen, du finnois makuu-vaunu (makuu = dormir, vaunu = wagon), du danois sove-vogn (sove $=$ dormir), etc. Le calque roumain vagon de dormit provient, très certainement, de la version allemande.

Une dualité identique de processus se répète à nouveau dans le composé anglais horse-power, l'unité de force adoptée par l'ingénieur écossais James Watt à la fin du XVIII' siècle. En 1838, nous trouvons déjà en français le calque " dévié " cheval-vapeur, forme base d'une vague terminologique internationale : caballo de vapor en espagnol, cavallo-vapore en italien, cavalo-vapor en portugais, cavall de vapor en catalan et cabalo de vapor en galicien. Par contre, les idiomes germaniques adopteront cette fois encore des calques littéraux de l'anglais : l'allemand Pferdestarke, le danois hestekraft, le hollandais pfaardekracht, etc. Le même schéma (cheval + force) a été adopté aussi en grec ( $\iota \pi \pi o \delta v \nu \alpha \mu \iota \xi)$ et jusqu'en basque, non sans une certaine surprise (zaldindar, de zaldi, cheval, et indar, force).

Dans le cas du mot américain skyscraper, cependant, le processus a été renversé. Le calque-traduction au français est ici littéral (gratte-ciel), d'où l'italien gratta-cielo, le 
catalan grata-cels, l'espagnol rascacielos, le portugais aranhaceus, et même le grec mo-

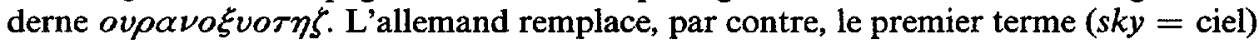
par le moins prétentieux Wolke, nuage, et obtient la forme Wolkenkratzer, formation que nous retrouverons en hollandais (wolkenkrabber). En suédois, l'apparent emprunt lexical skyskrapa, si proche de l'original anglais, est en fait un calque de l'allemand : sky $=$ nuage, $s k r a p a=$ gratter. Il en est de même en danois : skyskraber $(s k y=$ nuage, skrabe $=$ gratter) .

Les causes du nombre important de calques qui aujourd'hui circulent en espagnol, aussi bien qu'en italien ou en portugais, de même que celles de leur enracinement immédiat et parfois de leur internationalisation presque instantanée, sont bien évidentes, et de fait ne sont même pas linguistiques, mais strictement sociologiques : la présence croissante d'emprunts et de calques dans toutes les langues d'Occident répond en réalité à une forte convergence des modes de vie et de culture. La prolifération d'objets et de concepts communs est aujourd'hui plus grande que jamais, de même que plus grande leur diffusion. On peut difficilement qualifier la culture, la science ou la technique de ces quarante dernières années de françaises, d'anglaises ou d'allemandes, mais plutôt d'européennes ou d'occidentales, si en tout cas elles méritent un qualificatif de cette nature. Cette convergence vers une communauté occidentale d'idées est, de plus, renforcée par l'apogée des communications internationales, de plus en plus fréquentes et quotidiennes (y compris les agences de presse), par l'étude massive actuelle des langues étrangères et par les milliers de traductions qui nous entourent. (Qu'on se rappelle le chiffre que j'ai donné plus haut : plus de huit mille traductions sont sorties des imprimeries espagnoles seulement en 1985 ; mais il y a en outre, celles qui n'apparaissent pas dans l'ISBN : traductions de travail, résumés d'actes et de rapports de congrès, feuillets d'instruction pour le maniement d'appareils ménagers, les doublages cinématographiques et de télévision, les documents qu'avalisent les traducteurs assermentés, les traductions orales qu'effectuent les interprètes dans le monde entier...). C'est comme si une grande partie des langues occidentales s'acheminaient actuellement à pas accélérés vers une communauté terminologique internationale. Une fois abaissées les barrières conceptuelles qui peuvent séparer des nations d'autres nations, et alors que toutes participent, chaque jour davantage, de concepts, de notions, d'entités, de circonstances et de réalités inter et supranationales, il n'est en rien surprenant que les langues elles-mêmes reflètent, à travers la prolifération actuelle de calques, cette même " communion " internationale qui s'accroît de jour en jour. C'est là, la nouvelle force centripète qui peut arriver à contrecarrer la force centrifuge qui jusqu'ici a séparé des idiomes d'autres idiomes.

Répétés journellement, continuellement présents dans tous les moyens de communication oraux et écrits, les centaines de calques que compte aujourd'hui l'espagnol sont monnaie assez courante en 1986. Ce qui à son tour pourrait être appliqué dans les mêmes termes aux langues de son entourage historique et géographique. Nous vivons, et chaque jour nous le ferons davantage, dans une culture atlantique qui élimine peu à peu les compartiments étanches et crée des espaces communs. Frontières et langues ont atteint un haut degré de perméabilité, et les calques le démontrent. De même qu'ils démontrent que nous allons tout droit vers une "mentalité européenne", en termes qu'employa en 1956 B.E. Vidos, professeur de l'Université de Nimègue, "vers une unité linguistique et culturelle, un 'gemeineuropäischer Wortschatz' ».

Défenseur à outrance de la théorie de la convergence européenne et de la formation d'une terminologie générale paneuropéenne, Emilio Peruzzi commentait déjà en 1958, dans ses Saggi di Linguistica Europea publiés à Salamanque, que les imitations dans le domaine locutionnel, ou ce qui revient au même, les calques, car ils n'impliquent pas d'innovation dans les systèmes phonologique, morphologique et lexical des langues 
individuelles, parce qu'ils n'ont pas besoin d'adaptation, et ne doivent pas vaincre de résistances, sont plus importants encore pour la formation d'un "style européen " que les emprunts lexicaux. "Si comprende quindi come le convergenze degli idiomi europei nel campo locuzionale siano in numero infinito... »12 ; bien que, ajoute-t-il, ce soit là un phénomène qui, aux yeux d'un observateur peu averti, semble beaucoup moins manifeste, choquant et évident que celui des emprunts. Mais il n'y a pas de doute qu'à la longue il peut être aussi beaucoup plus définitif.

Dans la première circulaire que distribuèrent les organisateurs de cette réunion, on posait une question initiale qui consistait en même temps sa justification : " Pourquoi un colloque sur la fertilisation terminologique dans les langues romanes? "En plus de demander une réflexion multidisciplinaire sur le sujet, on ajoutait dans ces premières notes que " la confrontation des modes d'innovation terminologique ... présente plusieurs intérêts et pose les questions suivantes " :

Premièrement : Quels sont les types d'unités terminologiques qui s'imposent de facto dans les langues romanes à date récente? Selon ce qui a été exposé, ma réponse à une telle question est évidente : je suis fermement convaincu que les calques représentent ce type d'unité terminologique qui s'impose aujourd'hui.

Deuxièmement : Quels sont les types les plus propres à enrichir nos nomenclatures sans dénaturer chaque langue (grâce à une sorte de fertilisation) ? Il est évident, à nouveau, que ce sont les calques, pour les raisons qu'énumère Peruzzi, dérivées de leur condition de microtextes traduits.

Troisièmement : Quels sont les types d'unités terminologiques les plus généralisables au sein de la famille des langues romanes? La même réponse que la précédente : les calques. Et non seulement " généralisables ", mais déjà si "généralisés " que nos propres langues le reflètent.

Notes

1. Michel Chansou (1983) : "Calques et créations linguistiques", $M E T A, 22: 3$, p. 281.

2. Josip Jernej (1973) : "Traduzione e calco ", la Traduzione, Saggi e Studi, Trieste, Edizioni Lint, p. 253.

3. Valentín García-Yebra (1982) : Teoría y práctica de la traducción, Madrid, Editorial Gredos, p. 341.

4. Paris, Didier, 1977 (1958), p. 47.

5. "Le néologisme et nous", META, 18:1-2 (1973), p. 228.

6. Wolfram Wilss (1977) : The Science of Translation : Problems and Methods, Tübingen, Gunter Narr, p. 97.

7. p. 28 .

8. Barbara M. H. Strang (1970) : A History of English, London, Methuen \& Co. Ltd., 1977, p. 95.

9. Op. cit., p. 282 .

10. Op. cit., p. 95 .

11. De la première circulaire de ce colloque.

12. Emilio Peruzzi (1958) : Saggi di Linguistica Europea, Salamanca, C.S.I.C., Instituto Antonio de Nebrija (Tesis y Estudios Salmantinos, 10), p. 31.

APPENDICE

(Quelques calques espagnols de diverses langues et leurs équivalents français)

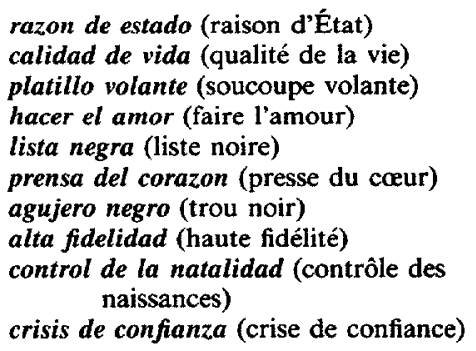

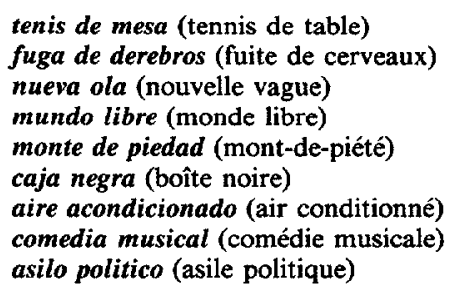

balanza comercial (balance commerciale) 
estacion de servicio (station-service) dama de honor (dame d'honneur) fuerzas armadas (forces armées) guerra fría (guerre froide) mesa redonda (table ronde) peloton de cabeza (peloton de tête) no violencia (non violence)

lluvia acida (pluie acide)

hora punta (heure de pointe)

interlocutor valido (interlocuteur valable)

librepensador (libre-penseur) materia prima (matière première) peso pluma (poids plume) plataforma (plate-forme) marea negra (marée noire) dar luz verde (donner le feu vert) grupo de presion (groupe de pression) huelga de celo (grève de zèle) llave de contacto (clef de contact) campaña electoral (campagne électorale) cara a cara (vis-à-vis)

banco de datos (banque de données) bestia negra (bête noire) caballo de vapor (cheval-vapeur) cadena de montaje (chaîne de montage) agua pesada (eau lourde)

lucha armada (lutte armée)

estado de sitio (état de siège)

tercer hombre (troisième homme)

tiempo libre (temps libre)

tienda de oxigeno (tente à oxygène)

novela negra (roman noir)

pleno empleo (plein emploi)

numero uno (numéro un)

jornada continua (journée continue)

hombre fuerte (homme fort) poderes publicos (pouvoirs publics) lucha por la vida (lutte pour la vie) fuerzas del orden (forces de l'ordre) contracultura (contre-culture)

fortaleza volante (forteresse volante) cabeza de lista (tête de liste)

derechos del hombre (droits de l'homme) enviado especial (envoyé spécial)

caza de brujas (chasse aux sorcières) casa publica (maison publique)

peso gallo (poids coq)

objetor de conciencia (objecteur de conscience)

enseñanza programada (enseignement programmé)

retrato robot (portrait-robot)

relaciones publicas (relations publiques)

telon de acero (rideau de fer)

punto de vista (point de vue)

reaccion en cadena (réaction en chaîne)

superhombre (surhomme)

conferencia de prensa (conférence de presse)

contraespionaje (contre-espionnage)

ciencia ficcion (science-fiction) café teatro (café-théâtre)

peso ligero (poids léger)

hombre rana (homme-grenouille)

jefe de estado (chef d'État)

largo-metraje (long métrage)

casa del pueblo (maison du peuple)

cascos azules (casques bleus)

crecimiento cero (croissance zéro)

escalada (escalade)

espionaje industrial (espionnage industriel)

zona franca (zone franche)

piel roja (peau rouge)

vision del munde (vision du monde)

universidad de verano (Université d'été)

trata de blancas (traite des blanches)

tierra batida (terre battue)

camara de comercio (chambre de commerce)

arreglo de cuentas (règlement de comptes)

derechos de autor (droits d'auteur)

camion cisterna (camion-citerne)

cortocircuito (court-circuit)

barrera del sonido (mur du son)

bienestar (bien-être)

dialogo de sordos (dialogue de sourds)

agencia de viajes (agence de voyages)

ala dura (aile dure)

tirar la esponja (jeter l'éponge)

madre de familia (mère de famille)

tercer mundo (tiers monde)

tercermundismo (tiers-mondisme)

tercemundista (tiers-mondiste)

opinion publica (opinion publique)

poner en escena (mettre en scène)

mayoria silenciosa (majorité silencieuse)

guerra relampago (guerre éclair)

mujer objeto (femme objet)

huida hacia adelante

goma de mascar (gomme à mâcher)

golpe de estado (coup d'État)

fabrica de sueñ (usine à rêves)

fotonovela (photo-roman)

linguistica aplicada (linguistique appliquée)

desobediencia civil (désobéissance civile)

dictadura del proletariado (dictature du prolétariat)

coexistencia pacifica (coexistence pacifique) musica de camara (musique de chambre)

balanza de pagos (balance de paiements)

ley marco (loi-cadre)

embotellamiento (embouteillage)

riñon artificial (rein artificiel)

sobre el terreno (sur le terrain)

puesta en escena (mise en scène)

rescacielos (gratte-ciel)

recursos naturales (ressources naturelles)

supermercado (supermarché)

canto del cisne (chant du cygne)

campo de concentracion (camp de concentration) ciudad dormitorio (cité-dortoir) 
coche cama (wagon-lit)

grandes almacenes (grands magasins)

mesa redonda (table ronde)

contra reloj (contre la montre)

pequeñas y medianas empresas (petites et moyennes entreprises)

revolucion cultural (révolution culturelle)

culo de saco (cul-de-sac)

carta abierta (lettre ouverte)

alta costura (haute couture)

marina mercante (marine marchande)

estar a la orden del dia (être à l'ordre du jour)

pabellon de caza (pavillon de chasse) esqui nautico (ski nautique)

cumbre (sommet)

camara de gas (chambre à gaz)

a nivel de (au niveau de)

tecnologia de punta (technologie de pointe) comandante en jefe (commandant en chef) mujer fatal (femme fatale)

misil tierra aire (missile sol-air)

la crema de la crema (la crème de la crème)

silla electrica (chaise électrique) 